\title{
On the accuracy of the independent column approximation in calculating the downward fluxes in the UVA, UVB, and PAR spectral ranges
}

\author{
Ronald Scheirer and Andreas Macke \\ Institute of Marine Research, Kiel, Germany
}

\begin{abstract}
In order to investigate the accuracy of simplification in modeling the radiative transfer in those solar spectral regions with major impacts on bio-organisms, i.e., the UVA $(0.32-0.4 \mu \mathrm{m})$, the UVB $(0.28-0.32 \mu \mathrm{m})$, and the photosynthetically active radiation (PAR, 0.4-0.7 $\mu \mathrm{m}$ ), radiative transfer calculations with varying treatments of cloud geometries (plane-parallel homogeneous (PPHOM), independent column approximation (ICA), and three-dimensional (3-D) inhomogeneous) have been performed. The complete sets of atmospheric information for 133 cloud realizations are taken from the three-dimensional nonhydrostatic mesoscale atmospheric model (GESIMA). A Monte Carlo radiative transfer model (GRIMALDI) has been developed that simulates scattering and absorption for arbitrarily three-dimensional distributions of cloud hydrometeors, air molecules, and water vapor. Results are shown for domain-averaged direct and total transmission (and so, implicitly, diffuse transmission) at the ground surface. In the UVA the PPHOM assumption leads to an underestimation in direct (total) downward flux by as much as $43(28) \mathrm{W} \mathrm{m}^{-2}$, which is about $49 \%$ (32\%) of the incoming irradiation, whereas results based on the ICA are almost identical to the 3-D case, except for convective clouds where the error in the UVA for direct (total) downward flux reaches $5(2) \mathrm{W} \mathrm{m}^{-2}$, or $6 \%(2 \%)$ of the incoming solar irradiation.
\end{abstract}

\section{Introduction}

The simplification of cloud geometries as plane-parallel homogeneous layers leads to well-known systematic errors in computations of solar cloud radiative fluxes, i.e., albedo, transmission, and absorption. Most applications of three-dimensional radiative transfer theory have focused on albedo or absorption [e.g., Chambers et al., 1997; Fu et al., 2000], while studies concerning the surface UV radiation used 1-D radiative transfer models [Charache et al., 1994] or were limited to few cloud realizations [Meerkötter and Degünther, 1999]. The present work investigates the effect of cloud inhomogeneity on total and direct transmission (and so, implicitly, on diffuse transmission) at the ground surface in the UVB (wavelengths of $0.28-0.32 \mu \mathrm{m}$ ), the UVA $(0.32-0.4 \mu \mathrm{m}$ ), and the photosynthetically active radiation (PAR, 0.4$0.7 \mu \mathrm{m}$ ), the solar spectral bands with the major impact on bio-organisms.

The three-dimensional radiative transfer in inhomogeneous cloudy atmospheres is treated by means of a direct Monte Carlo approach which is applied to modeled cloud fields, consisting of three-dimensional inhomoge-

Copyright 2001 by the American Geophysical Union.

Paper number 2001JD900130.

0148-0227/01/2001JD900130\$09.00 neous cloud structures. The microphysical and macrophysical properties of the three-dimensional clouds used in this study are described in section 2 . Section 3 specifies how scattering and absorption at atmospheric gases is taken into account. The Monte Carlo radiative transfer model is described in section 4 , followed by a discussion of the results in section 5 . Section 6 provides a summary and conclusion.

\section{Clouds}

The spatial cloud fields under investigation are calculated by means of the three-dimensional, nonhydrostatic mesoscale atmospheric model (GESIMA) [Eppel et al., 1998; Hagedorn, 1996].

Cloud microphysical processes in GESIMA are based on a bulkparameterization by Levkov et al. [1992]. The spatially, resolved GESIMA data that are required for the radiative transfer calculations are temperature, pressure, water vapor content, water content for liquid water, snow, ice, and rain, as well as the number concentrations of these hydrometeors. These data are transformed to volume extinction coefficients and effective particle sizes as described by Macke et al. [1999].

GESIMA calculations are forced by adding a local water vapor excess of variable size to the initially horizontally homogeneous atmosphere. The (thermo-) dy- 

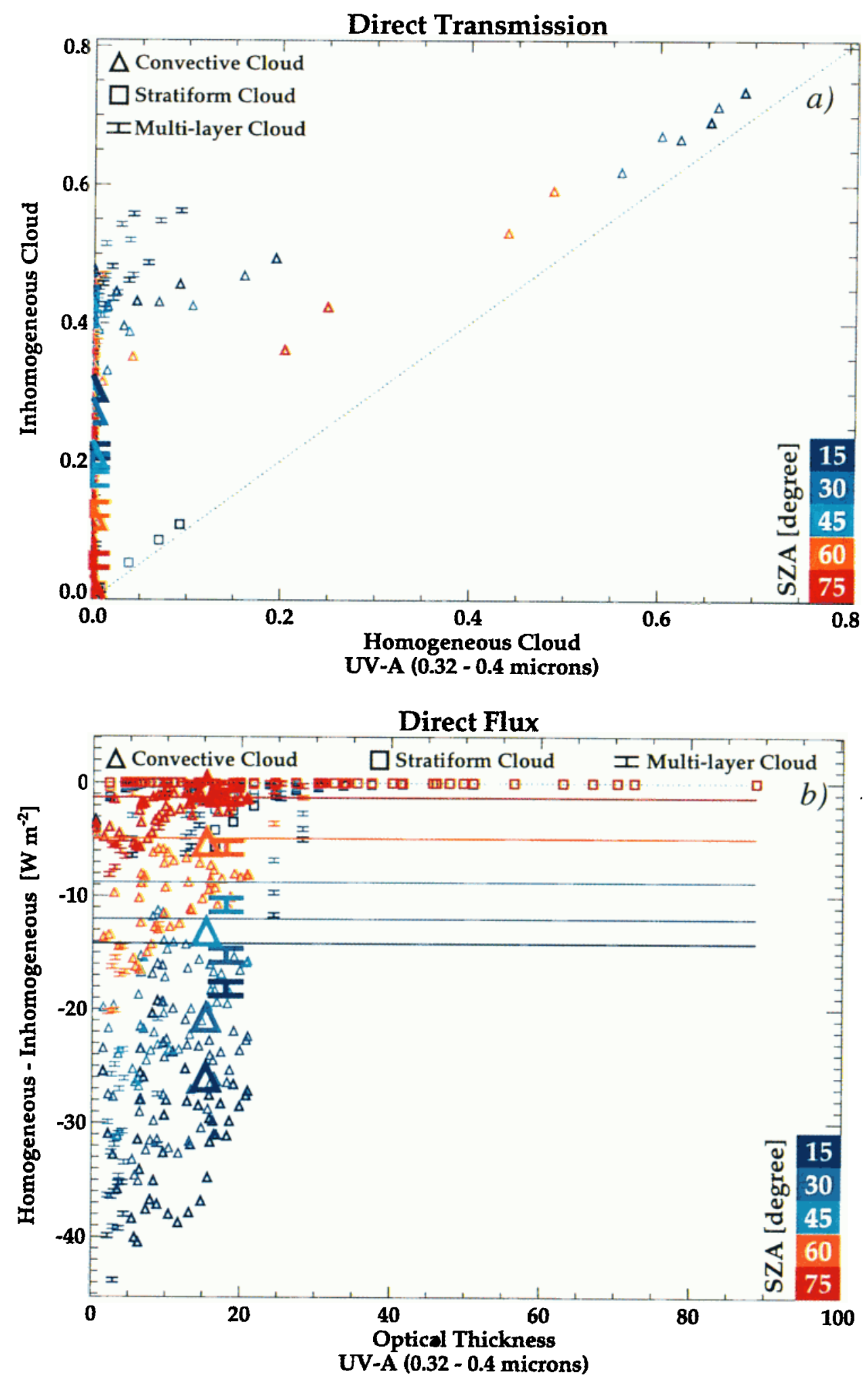

Plate 1. (a) Scatterplot of direct transmissions and (b) direct downward flux differences versus domain-averaged optical thickness for horizontal homogeneous clouds and 3-D clouds in the UVA. The colored lines (Plate 1b) mark the mean differences for the corresponding SZA. Large symbols denote clouds used in a detailed examination. 


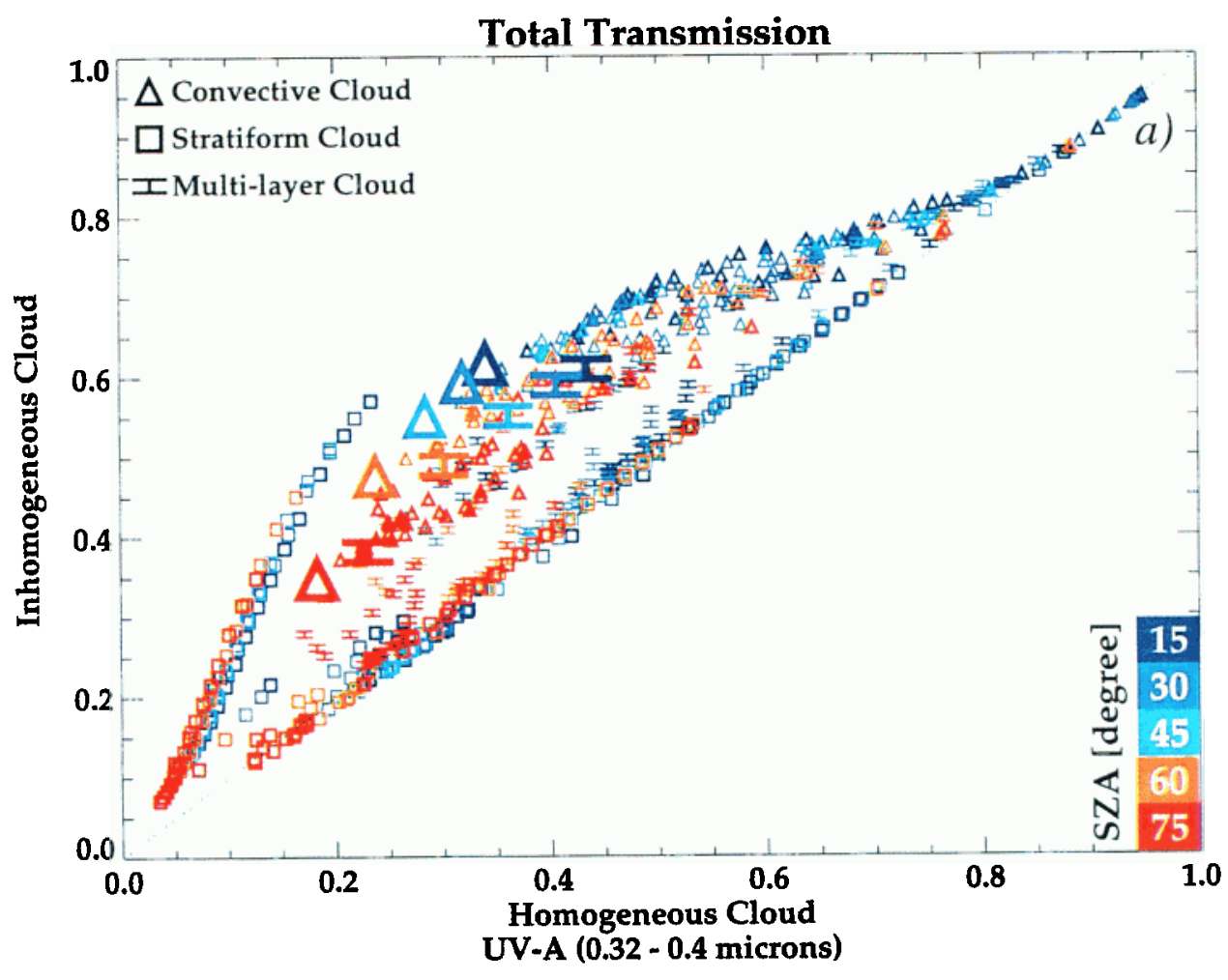

Total Downward Flux

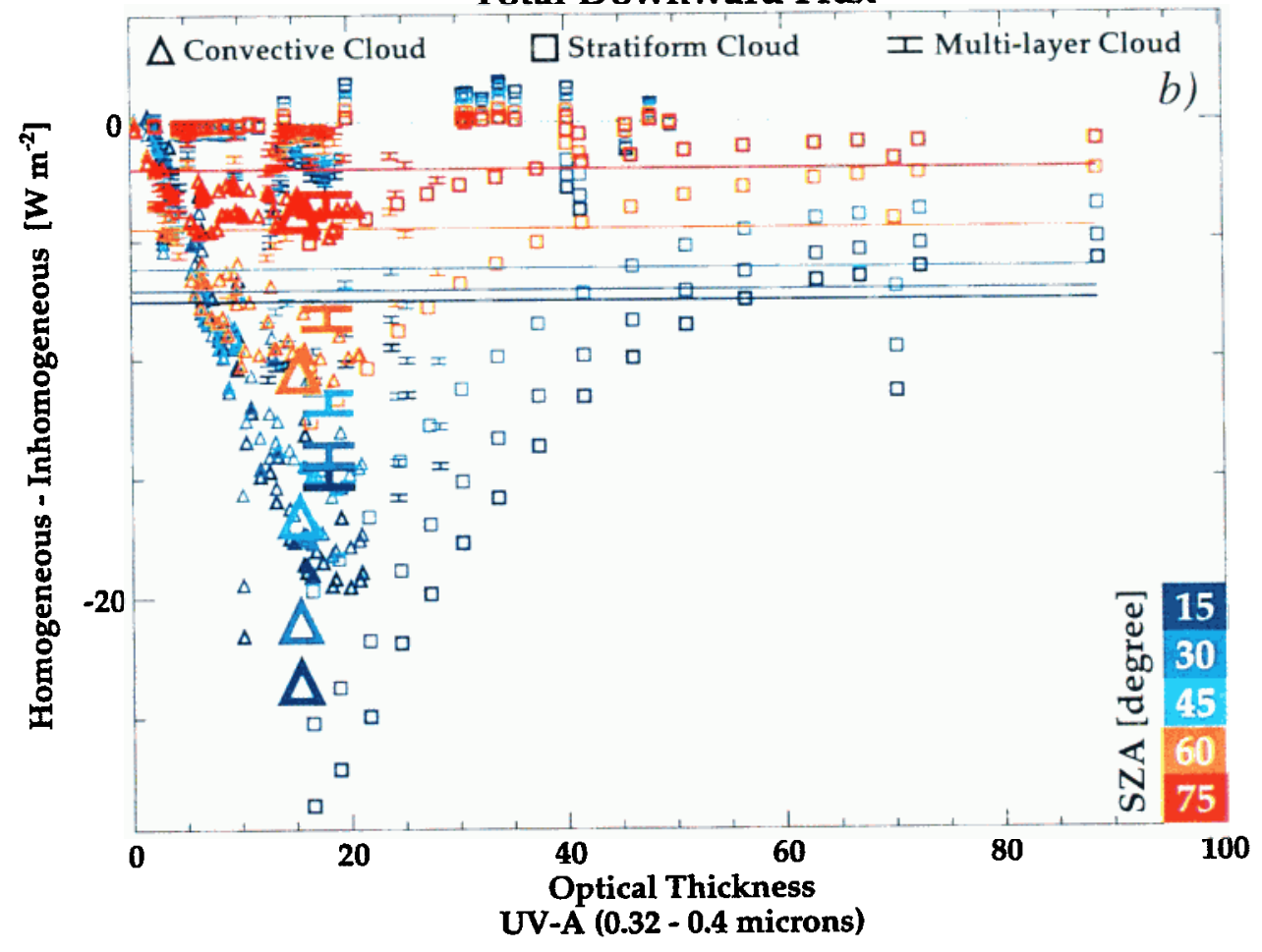

Plate 2. Same as Plate 1, but for total transmission and total downward flux. 

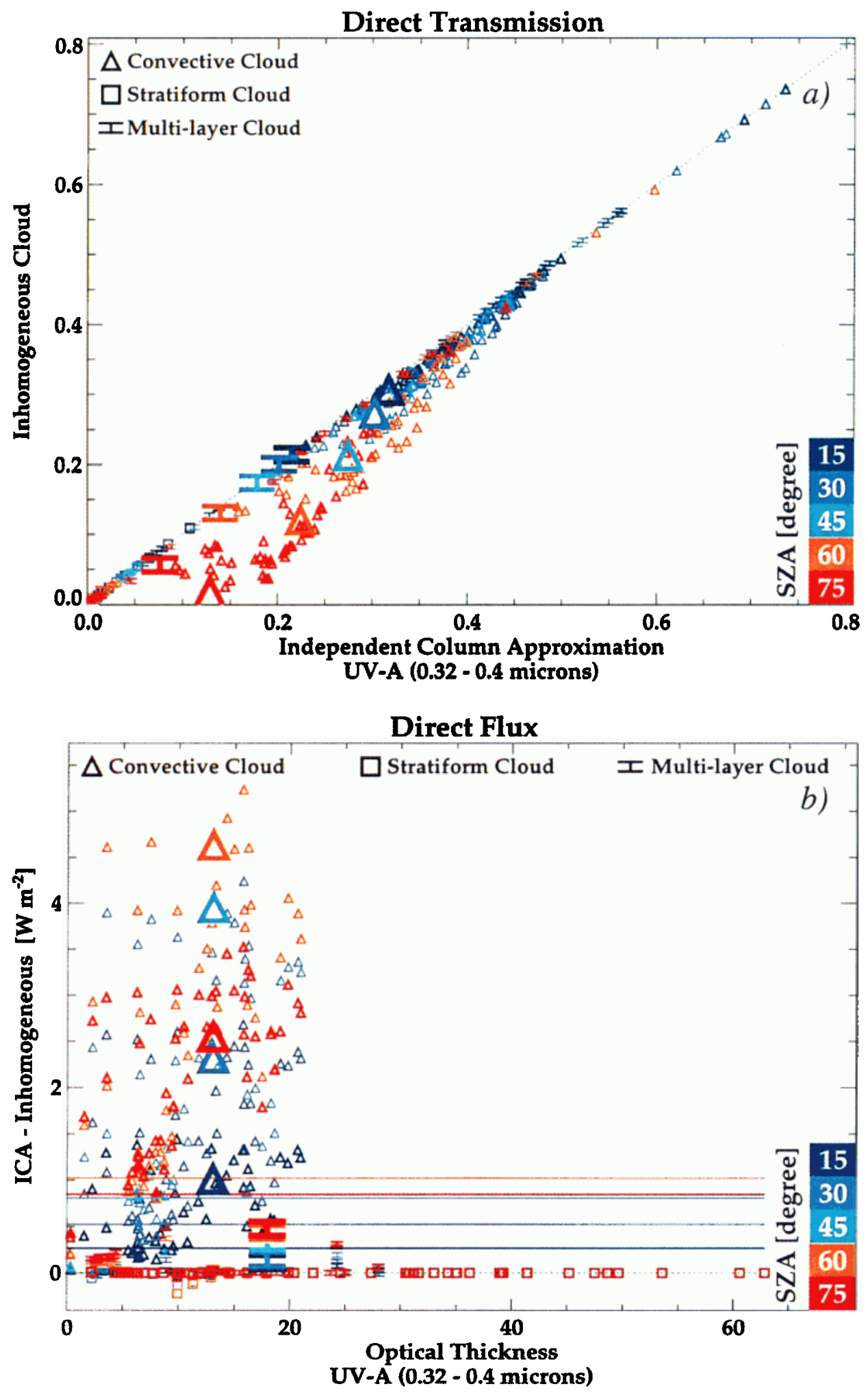

Plate 3. Same as Plate 1, but for the comparison between ICA and 3-D clouds. 
namical cloud formation processes resulting from this induced instability yield a 3-D inhomogeneous atmospheric structure. The calculations are stopped as soon as the cloud exceeds the model domain.

Out of 133 cloud realizations, 46 are stratiform, 50 are convective (e.g., Figure 1a), and 37 have separate layers. The stratiform and convective clouds are distinguished by the way the local water vapor excess is induced into the model domain. Multilayer clouds are simply identified by one or more cloud-free levels.

For the Monte Carlo radiative transfer calculations the model domain is reduced to the smallest right parallelepiped that embraces cloud top and cloud sides. The lower bound of the model domain is set to the ground surface in order to get the downwelling radiative fluxes and transmissivities at the ground.

\subsection{Stratiform and Convective Clouds}

The GESIMA cloud realizations for stratiform and convective clouds are initialized with five temperature and humidity profiles, taken from radiosonde measurements over the Baltic Sea area during summer 1995 and 1996, over the South Pacific in February 1995, and over the North Atlantic during January and July 1989 [Hagedorn, 1996]. The model runs for roughly 2 hours with a time step of $5 \mathrm{~min}$, so that a maximum of 25 cloud realizations result from each profile. In order to avoid finite size effects, the GESIMA calculations are terminated as soon as the cloud field exceeds the model domain. The latter ranges from the ground to $10-\mathrm{km}$ height vertically; and $21 \mathrm{~km}$ by $21 \mathrm{~km}$ horizontally. The vertical resolution varies from $100 \mathrm{~m}$ at the ground to $1 \mathrm{~km}$ at the top. The horizontal resolution is $1 \mathrm{~km}$.

\subsection{Multilayer Clouds}

Clouds with separated layers are produced by initializing GESIMA with four radiosonde measurements obtained between $50^{\circ} \mathrm{N}$ and $70^{\circ} \mathrm{N}$ during July and August 1989. A model integration time of about 2 hours and a time step of $10 \mathrm{~min}$ provides up to 13 cloud realizations for each radiosonde profile. The spatial dimensions are $104 \mathrm{~km}$ by $104 \mathrm{~km}$ horizontally and $10 \mathrm{~km}$ vertically with a horizontal resolution of $2 \mathrm{~km}$ and the same vertical resolution as that for the stratiform and the convective clouds.

\section{Radiative Properties of Atmospheric Gases and Hydrometeors}

Absorption by gas molecules (water vapor, oxygen, and carbon dioxide) is expressed in terms of their single scattering albedo $\left(\omega_{0}\right)$. Depending on the mean profiles of temperature, pressure, and absorber amount for each of the nine cloud series described above, the profiles of effective transmissivity with regard to the gas absorption are calculated line by line for the spectral intervals listed in Table 1. This is to avoid the computation of unrealistic high effective absorption coefficients due to
Table 1. Wavelength Ranges, Center Wavelength, and Solar Irradiation for Each Solar Spectral Band Calculated From the Data Set Given by Thekaekara [1974]

\begin{tabular}{lccc}
\hline Name & Range, $\mu \mathrm{m}$ & $\lambda, \mu \mathrm{m}$ & Irradiation, $\mathrm{W} / \mathrm{m}^{2}$ \\
\hline UVB & $0.28-0.32$ & 0.30 & 22.39 \\
UVA & $0.32-0.40$ & 0.36 & 88.03 \\
PAR & $0.40-0.70$ & 0.55 & 51623 \\
\hline
\end{tabular}

supersaturated lines. Molecular spectral line data are taken from the HITRAN database [Rothman et al., 1987]. Transmissivities are transformed into molecular absorption coefficients by taking the vertical depth and molecular number density of each model layer into account. Together with the Rayleigh scattering coeffcient the single scattering albedo can then readily be obtained. A detailed description of this procedure is given by Scheirer and Macke [2000].

The inhomogeneous distribution of the absorption coefficient is realized by multiplying the absorber density of each GESIMA grid box with the molecular absorption coefficient from the precalculated profile.

The Rayleigh scattering cross section $\sigma$ is obtained from an analytic formula given by Bucholtz [1995].

The Rayleigh phase function given by Chandrasekhar [1960] is used to calculate the new photon direction after a photon-molecule interaction.

The wavelength dependent depolarization factor needed for the correction term in this phase function is fitted to the data given by Bucholtz [1995].

Absorption and scattering properties of cloud particles are obtained from Mie calculations for water droplets and from ray-tracing calculations for nonspherical snow, rain, and ice particles [Macke et al., 1999].

The total phase function for each grid box is obtained by averaging the Rayleigh scattering phase function and the scattering phase function of the hydrometeors weighted by their individual scattering cross sections. Similarly, the total single scattering albedo is obtained from the sum of the Rayleigh scattering coefficient and the scattering coefficient of the hydrometeors, divided by the total extinction coefficient.

\section{Monte Carlo Radiative Transfer}

In order to obtain the solar broadband upwelling and downwelling radiative fluxes, a Monte Carlo radiative transfer model (GRIMALDI), based on the model by Macke et al. [1997], has been developed.

The influence of different cloud aspect ratios (ratio of cloud vertical to horizontal dimension) is minimized by applying horizontally reflecting boundary conditions to the photon paths. The entry point for each incoming photon is randomly distributed along the upper boundary of the model domain. The solar azimuth angle is also randomly chosen. The surface is assumed to be black. 
Each model grid box is characterized by its position, geometrical dimensions, extinction coefficient, scattering phase function, and single scattering albedo.

Photons are traced with a direct Monte Carlo simulation as described by Macke et al. [1999]. Additionally, absorption is taken into account by multiplying the initial photon weight with the total single scattering albedo for each scattering event. Sensitivity studies have shown that $2 \cdot 10^{6}$ photons suffice to obtain statistically stable domain-averaged radiative fluxes. For reliable calculations of net horizontal transports at each cloud model grid cell, $2 \cdot 10^{8}$ incoming photons have been used.

The radiative transfer calculations have been performed for five solar zenith angles (SZA) $\left(15^{\circ}, 30^{\circ}, 45^{\circ}\right.$, $60^{\circ}$, and $75^{\circ}$ ) and for the following cloud geometries: (1) three-dimensional inhomogeneous clouds with reflecting horizontal boundary conditions (3-D); (2) column-bycolumn horizontally homogeneous clouds (independent column approximation (ICA)); (3) domain-averaged horizontally homogeneous clouds (plane-parallel homogeneous (PPHOM)), a special case of the ICA.

This results in a total of 1995 experiments for each spectral band (133 clouds, $5 \mathrm{SZA}$, and 3 different cases (3-D, ICA, and PPHOM)). On a DEC Alpha workstation (AXP533 AU2) the calculation of $2 \cdot 10^{6}$ photons ranges from $4 \mathrm{~min}$ to $90 \mathrm{~min}$ depending on the cloud optical thickness and SZA.

The $3-\mathrm{D}$ case represents the most realistic cloud geometry. It is used to check the accuracy of the two approximations. The ICA neglects horizontal photon transport between adjacent columns. Fluxes, calculated with this assumption, result from a sum of onedimensional radiative transfer computations. The column foot point varies between $1 \times 1 \mathrm{~km}^{2}$ (convective and stratiform clouds) and $2 \times 2 \mathrm{~km}^{2}$ (multilayer clouds). The PPHOM approximation is implemented by averaging the optical properties for each layer of the model domain and setting all comprehended model boxes to these mean values. This leads to a horizontally homogeneous optical thickness which is equal to the mean optical thickness of the 3-D cloud.

\section{Results and Discussion}

Radiative fluxes for the three spectral bands (UVA, UVB, PAR) are basically determined by the scattering at the cloud particles and only to a small extent by Rayleigh scattering and molecular absorption. We found that owing to the similar scattering properties of the atmospheric hydrometeors in these three spectral bands, the resulting fluxes show very similar behavior in each band. Consequently, the following discussion focuses on UVA only.

In order to localize characteristic errors due to the simplified treatment of the radiative transfer problem (PPHOM, ICA), Plates 1-4 show scatterplots (3-D versus PPHOM and ICA: upper diagram in each plate) and flux differences versus optical thickness (PPHOM and ICA minus 3-D: lower diagram in each plate). Cloud types (convective, stratiform, multilayered) are distinguished by different symbols, and solar zenith angles, by different colors. The terms "underestimation" and "overestimation" used in the following are defined with respect to the $3-\mathrm{D}$ results.

Plate 1 compares direct transmission and direct downward flux at the ground surface for $3-\mathrm{D}$ and PPHOM clouds. In general, the simplification of horizontally homogeneous clouds leads to an underestimation of the direct transmission mainly because of the exponential dependency of direct transmission on cloud optical thickness. This nonlinearity is most pronounced for small and moderate cloud optical thickness. Largest deviations are found for the most inhomogeneous convective and multilayered clouds. The deviations increase with decreasing SZA. In contrast to the 3 -D cases, a large number of homogeneous clouds show no direct transmission at all. This is simply due to the lack of cloud holes which, in the 3-D cases, allow for direct transmission even for optically thick clouds.

The direct flux differences shown in the lower diagram reach $44 \mathrm{~W} \mathrm{~m}^{-2}$, which is about $50 \%$ of the total incoming solar flux in this spectral region (see Table 1). Largest differences occur for convective and multilayered clouds almost independent of optical thickness. There is a slight tendency that the direct flux differences decrease with increasing $\tau$ because of the almost linear albedo $(\tau)$ relationship at large optical thickness. This also explains the decreasing mean direct flux differences (horizontal lines) with increasing solar zenith angle, i.e., increasing effective optical thickness. For a mean solar zenith angle of $45^{\circ}$ the average difference in the direct flux is about $9 \mathrm{~W} \mathrm{~m}^{-2}$.

Results for total transmission and total downward flux are shown in Plate 2. In general, homogeneous clouds underestimate the total transmission strongest for moderate transmissions, i.e., where the nonlinearity in the transmission versus optical thickness relationship is strongest. Large differences are also found at small transmissions for large solar zenith angles and at large transmissions for small solar zenith angles, i.e., tor regions with moderate effective transmissions. In most cases, the homogeneous cloud assumption underestimates total transmission strongest for convective clouds. For stratiform clouds, the differences are negligible except for one series of cloud realizations with total transmissions between 0.05 and 0.21 . These clouds feature a small mean effective size of ice particles and a large optical thickness. They may be described as thick cirrostratus clouds with a correspondingly high albedo. Optical thinner parts (3-D case) in this type of cloud can enhance the tranmission in contrast to the PPHOM case very effectively.

As is shown in Plate 2b, largest differences in total downward flux occur at domain-averaged optical thickness values around 10 . Homogeneous clouds underes- 

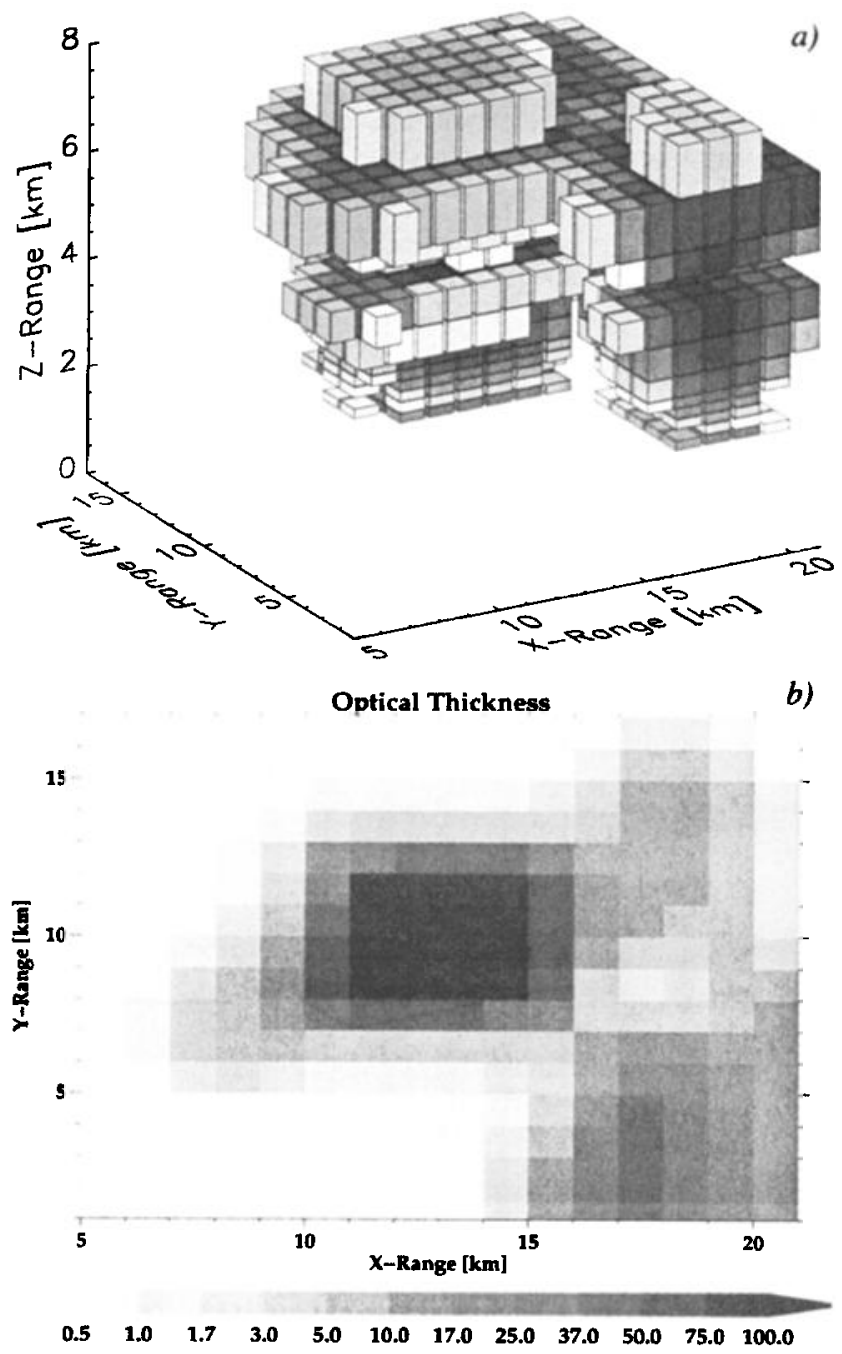

Figure 1. (a) Shape and (b) optical thickness of the cloud, used for the detailed examination of the influence of spatial resolution and solar zenith angle on the accuracy of the ICA.

timate the flux by as large as $30 \mathrm{~W} \mathrm{~m}^{-2}$. Averaging along optical thickness leads to a mean underestimation of about $8 \mathrm{~W} \mathrm{~m}^{-2}$ for $\mathrm{SZA}$ of $15^{\circ}$ and $30^{\circ}$. The decrease of flux differences with increasing SZA is mainly caused by the reduced incoming energy at a lower Sun elevation. The transmission is nearly independent on the SZA. This insensitivity is caused by multiple scattering. For the reverse reason, the direct downward flux as shown in Plate 1b strongly depends on the solar zenith angle.

The ICA is often considered a sufficiently accurate approximation for calculating domain-averaged solar radiative fluxes for inhomogeneous cloud fields [e.g., $\mathrm{Ca}$ halan et al., 1994]. Plate 3 shows direct transmission and direct downward flux resulting from the 3-D calculations and the ICA. Largest differences are found for convective clouds at small transmissions and for large solar zenith angles. Contrary to the homogeneous cloud assumption shown in Plate 1, the ICA overestimates the direct transmission. The independent treatment of each atmospheric column allows for large transmission for optically thin columns, whereas for the 3-D case photons are horizontally transported into regions of larger optical thickness and thus have a smaller probability for direct transmission. Obviously, this mechanism is strongest for large solar zenith angles.

The differences in direct downward fluxes exceed 5 $\mathrm{W} \mathrm{m} \mathrm{m}^{-2}$ for some convective cloud realizations at large solar zenith angles. The mean differences increase from $0.2 \mathrm{~W} \mathrm{~m}^{-2}$ at a solar zenith angle of $15^{\circ}$ to $1 \mathrm{~W} \mathrm{~m}^{-2}$ at $60^{\circ}$.

Plate 4 shows the total transmission and the total downward flux for the 3-D case and the ICA. Except for some convective clouds at large and small solar zenith angles, the domain-averaged total transmission is excellently estimated by the ICA. Largest differences in the total downward flux are about $0.9 \mathrm{~W} \mathrm{~m}^{-2}$. However, averaging over all clouds with the same solar zenith angle provides a maximum mean underestimation of 0.4 $\mathrm{W} \mathrm{m} \mathrm{m}^{-2}$ at a solar zenith angle of $15^{\circ}$ and a maximum mean overestimation of $0.4 \mathrm{~W} \mathrm{~m}^{-2}$ at a solar zenith angle of $75^{\circ}$.

We note that whereas the ICA overestimates the direct downward flux, it underestimates the total downward flux at small solar zenith angles, which implies a strong underestimation of the diffuse downward flux. Therefore for applications which are sensitive to the ratio of diffuse to direct irradiation at the ground, the ICA may provide large errors. However, in comparison with the systematic errors of the PPHOM assumption shown in Plates 1 and 2, the ICA yields considerably more accurate results for domain-averaged direct and diffuse transmissions.

It is obvious that the ICA works best for cloud fields with little horizontal variations. However, with decreasing horizontal variability, the results for the ICA and the PPHOM approximation converge so that there is no need for the ICA anymore. The delicate problem with the ICA is that the column size should be small enough to resolve the actual horizontal cloud structure, and it must be large enough to ensure that each column can be treated independently, i.e., that horizontal transports between adjacent columns can be neglected. The influence of horizontal resolution on the radiative fluxes as determined by the ICA is investigated exemplarily for one single convective cloud (marked by big triangles in Plates 1 to 4). The spatial structure and the horizontal optical thickness distribution of this specific cloud is shown in Figure 1. Three-dimensional and ICA radiative transfer calculations have been performed for five SZAs with a horizontal resolution of $1 \times 1 \mathrm{~km}^{2}$ (original resolution, in the following denoted as "truth"), $2 \times 2 \mathrm{~km}^{2}, 4 \times 4 \mathrm{~km}^{2}, 8 \times 8 \mathrm{~km}^{2}$, and $16 \times 16 \mathrm{~km}^{2}$ (completely horizontally homogeneous, PPHOM case). Differences (ICA - truth (percent)) in direct, diffuse, and total transmission and in absorption as a function of zenith angle and horizontal resolution are shown in Figure 2. 

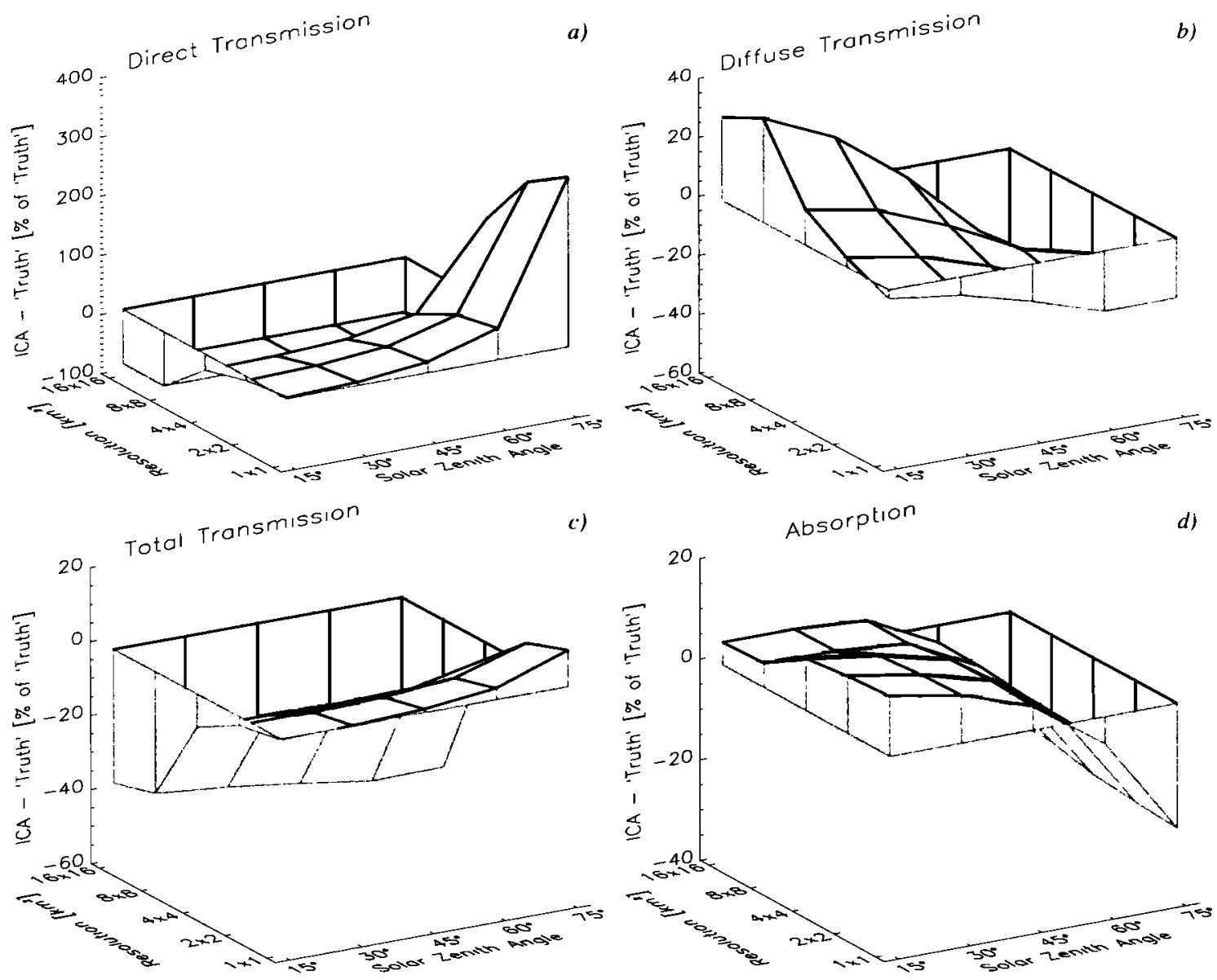

Figure 2. Error of the ICA in percent of "truth" (3-D Monte Carlo run with a resolution of $1 \times 1 \mathrm{~km}^{2}$ ) for (a) direct transmission, (b) diffuse transmission, (c) total transmission, and (d) absorption as a function of resolution and SZA.

With decreasing resolution the "true" direct transmission (Figure 2a) is more and more underestimated by the ICA because regions of small optical thickness are no longer resolved. This is a direct effect of the exponential dependency of direct transmission on optical thickness. For only moderate to large SZA and horizontal column dimensions smaller than $4 \mathrm{~km}$, the ICA overestimates the direct transmission. For this region the almost horizontal incoming photon directions reduce the likelihood for direct transmission through local regions of small optical thickness in the 3-D calculations. In general, the ICA underestimates direct transmission for column sizes larger than $4 \mathrm{~km}$ by about $100 \%$.

For diffuse transmission (Figure $2 \mathrm{~b}$ ), the relative errors of the ICA strongly depend on both spatial resolution and SZA. In general, the ICA results are becoming smaller with increasing resolution and with increasing SZA, with an overestimation of the true results at small resolutions and small SZA and an underestimation everywhere else. The overestimation is due to the increased smoothening of the cloud structure which increases the contribution of diffuse transmission at the expense of direct transmission. Again, for large SZA, horizontal transports into regions of large optical thickness are not accounted for in the ICA calculations, and the diffuse transmission is smaller than that for the 3-D case.

The overall errors in direct plus diffuse transmission are shown in Figure 2c. Although the individual errors in direct and diffuse transmission partly cancel out, there remains a general underestimation of the total transmission which is strongest for large ICA column sizes. The gradient in the relative errors of the ICA total transmission is strongest at a column size of $4 \mathrm{~km}$, which indicates that at least a $2-\mathrm{km}$ resolution should be used for ICA calculations. Again, we note that increasing the ICA resolution beyond $1 \mathrm{~km}$ will not continue to reduce the ICA errors because of the large contribution of horizontal transports for each individual column.

The absorption (Figure 2d) is overestimated by the ICA for solar zenith angles from $15^{\circ}$ to $45^{\circ}$ and seems to be nearly insensitive to changes in resolution. A further increase in SZA results in an underestimation with largest differences occurring at $75^{\circ}$. This is the consequence of underestimating the diffuse transmission for large SZA.

The contribution of net horizontal transports $H(x, y)$, 

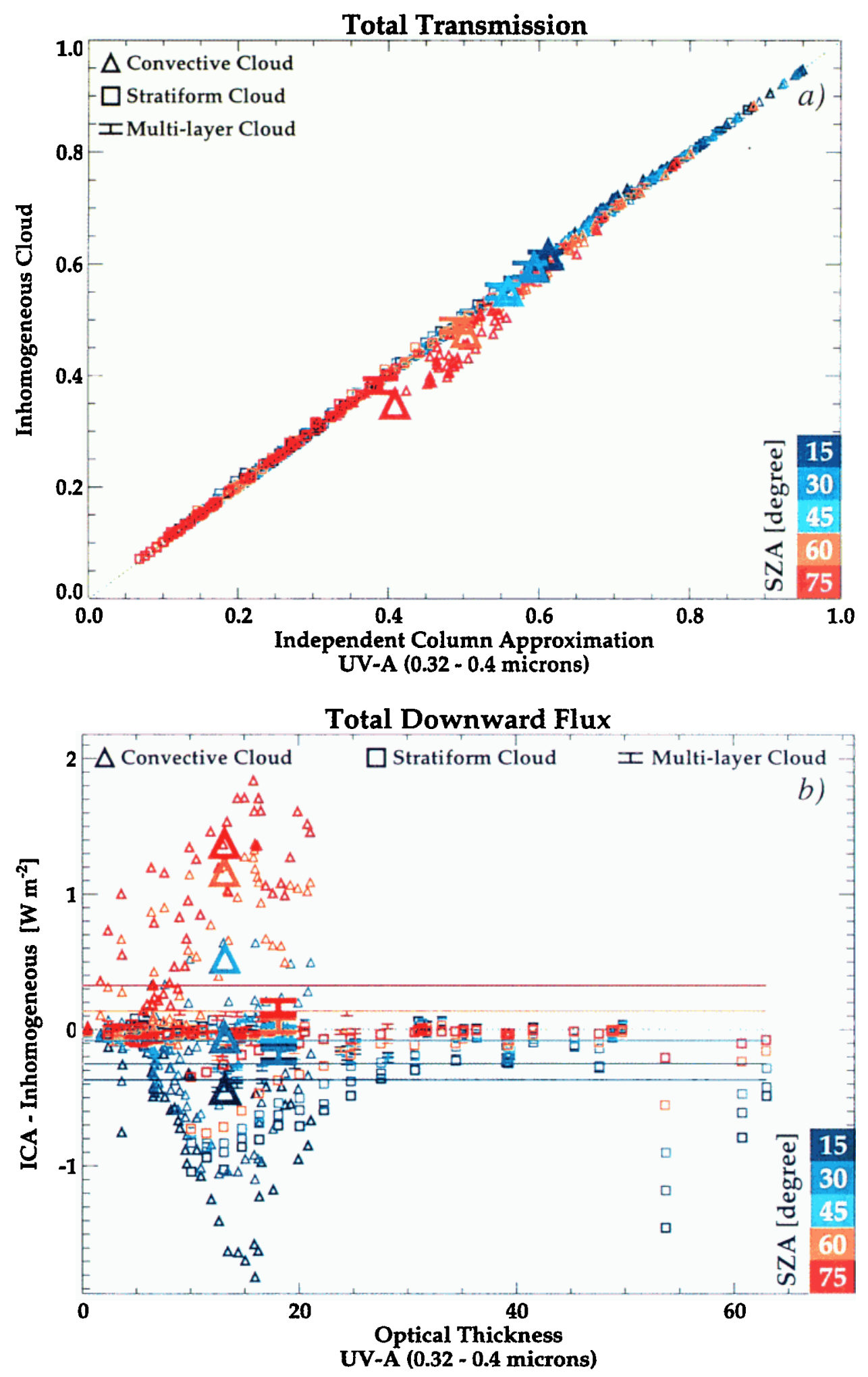

Plate 4. Same as Plate 3, but for total transmission and total downward flux. 

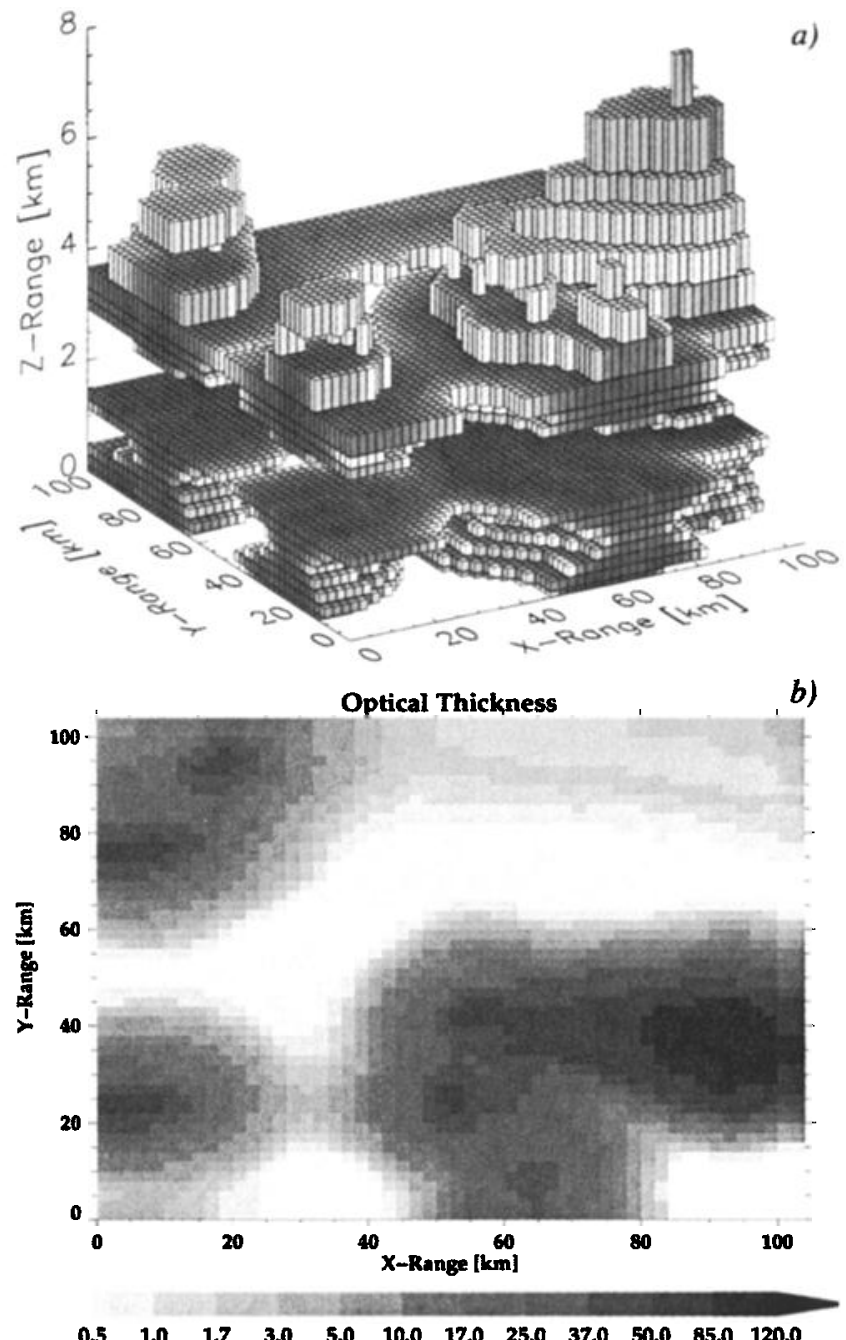

Figure 3. (a) Three-dimensional shape and (b) horizontal distribution of optical thickness of the cloud, used for the detailed investigation of the changes in horizontal transport due to changes in SZA.

defined as

$$
H(x, y)=1-R(x, y)-T(x, y)-A(x, y),
$$

where $R, T$, and $A$ denote albedo, total transmission, and absorption at a horizontal grid box $(x, y)$, is studied in more detail exemplarily for a multilayered cloud. A multilayered cloud is chosen because of its large horizontal dimension and thus for low influences of border effects. The domain-averaged radiative fluxes for this cloud are marked with large "horizontal $\mathrm{H}$ " symbols in Plates 1-4. Spatial structure and horizontal optical thickness distribution are shown in Figure 3. The horizontal distributions of net horizontal transport for SZA of $15^{\circ}, 30^{\circ}, 60^{\circ}$, and $75^{\circ}$ are presented in Plate 5. Negative values correspond to a net loss of photon energy; positive values, to a gain of photon energy. Note that absorption is very small at the spectral region under investigation. Therefore $H$ is basically determined by scattering. In general, a net horizontal transport at a given column occurs if the number of photons that are entering this column from top or side does not equal the number of photons that are leaving the column. The enhanced loss of photons from border columns with large optical thickness results from an artificial shadowing effect of reflecting boundary conditions.

For low SZA (Plates 5a and 5b), columns with large optical thickness lose photons to columns with lower optical thickness, which applies eminently to the regions with a large gradient in optical thickness. Solar photons that are entering optically thin regions are transmitted toward the ground within the same region and do not contribute to the local net horizontal transport. On the other hand, photons that are entering regions of large optical thickness are scattered into optically thin regions where they can escape more easily toward the ground or to space.

For large SZA (Plate 5d) the situation is reversed. The incoming solar photons are almost horizontally passing regions of small optical thickness without contributing to the local net horizontal transport. As soon as the photons enter optically thick regions, a large portion is scattered toward the ground or back to space. Thus for large SZA, optically thick regions gain photons at the expense of optically thin regions. Since the local gain and loss of photons changes sign when going from small to large SZA, it follows that there must be an SZA for which the local net horizontal transport is close to zero. For the clouds used in this study, this is the case for SZA of around $60^{\circ}$ as illustrated in Plate $5 \mathrm{~d})$.

The amount of net horizontal transport is a measure of the applicability of the ICA. Therefore Plate 5 shows that the ICA is most unreliable for extreme SZA in regions of strong gradients of cloud optical thickness. Here the net horizontal transport can be as large as \pm 0.3 , which implies that almost one third of the incoming energy is effectively distributed into adjacent columns. Again, we note that with decreasing resolution, the ICA will provide less net horizontal transport, but at the same time the domain-averaged radiative fluxes will deviate more from the true 3-D case.

\section{Conclusions}

The assumption of horizontally homogeneous clouds leads to large errors in calculating the transmitted solar energy in the UVA, UVB, and PAR, particularly for the ratio of direct to diffuse transmission. Since the penetration depth of solar irradiation into the ocean body or into canopy is a directional property, the PPHOM assumption may provide considerably wrong amounts of light supply. In general, the PPHOM assumption underestimates the total transmission with largest errors at low SZA, i.e., where the incoming solar energy is largest.

The ICA provides considerably better results for the domain-averaged radiative fluxes. However, for convec- 

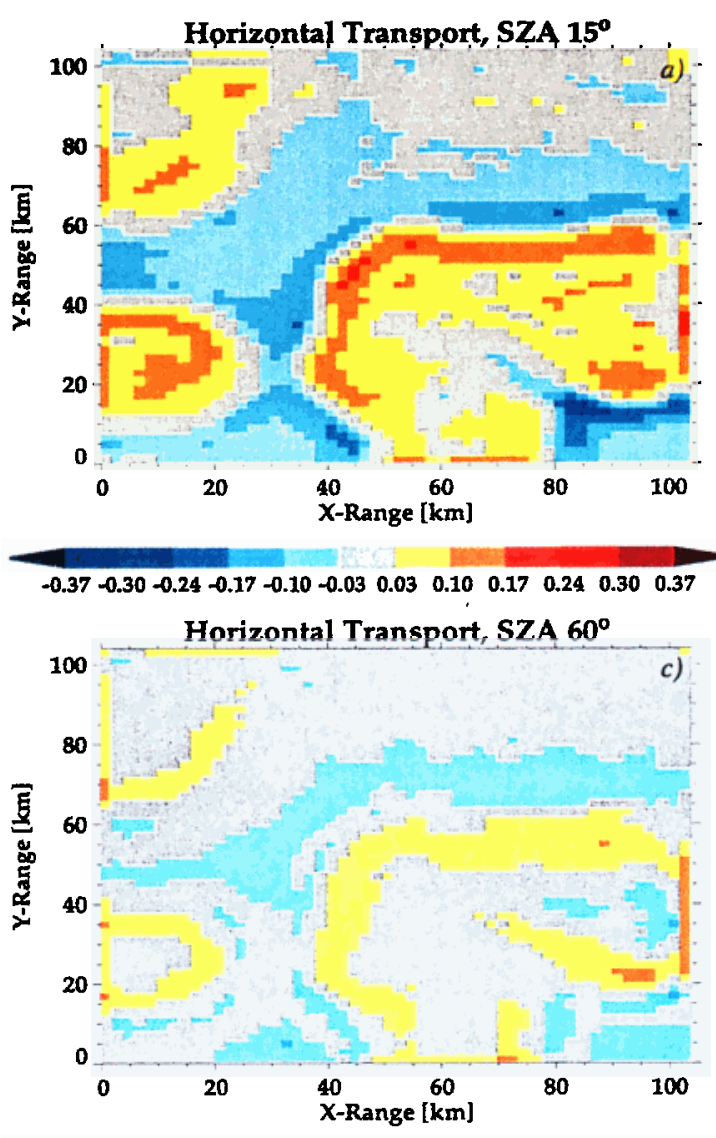

$\begin{array}{lllllllllllllllllllllllllll}-0.37 & -0.30 & -0.24 & -0.17 & -0.10 & -0.03 & 0.03 & 0.10 & 0.17 & 0.24 & 0.30 & 0.37\end{array}$
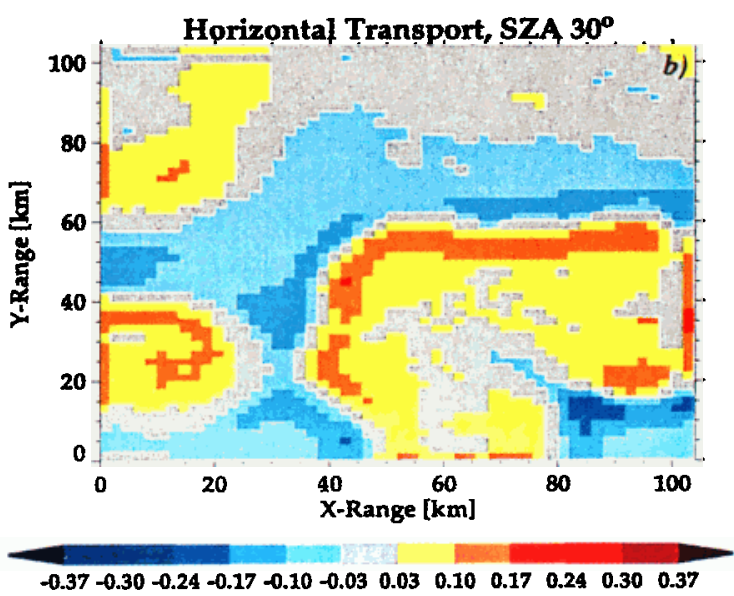

Horizontal Transport. SZA $7^{\circ}$

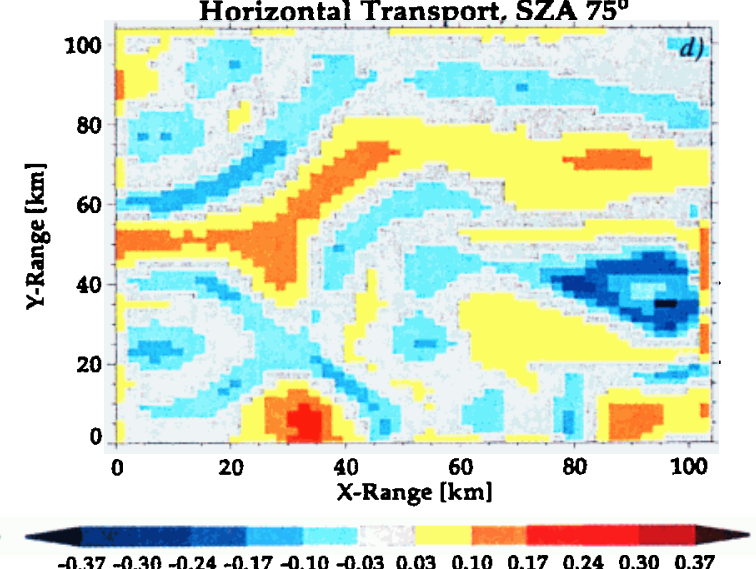

$\begin{array}{llllllllllll}-0.37 & -0.30 & -0.24 & -0.17 & -0.10 & -0.03 & 0.03 & 0.10 & 0.17 & 0.24 & 0.30 & 0.37\end{array}$

Plate 5. Horizontal transports for different SZA: (a) $15^{\circ}$, (b) $30^{\circ}$, (c) $60^{\circ}$, and (d) $75^{\circ}$. Negative values denote transports into the box.

tive clouds direct (total) transmission is overestimated (underestimated) for low solar zenith angles, while both direct and total transmission are overestimated at large SZA, i.e., where the incoming solar energy is small. Thus from an energetic point of view, it appears that the ICA is a reasonable approximation of the 3-D radiative transfer problem for domain-averaged radiative fluxes. This has been shown earlier for artificially constructed [Cahalan et al., 1994] and two-dimensional [Fu et al., 2000] cloud fields and is now confirmed for the more physically based mixed phase 3 -D cloud fields used in this study.

If the ICA does not capture the original resolution of a 3-D cloud field, it produces erroneous radiative fluxes which are, in terms of magnitude and sign, very sensitive to both the solar zenith angle and the applied horizontal resolution. Note that these results are based on calculations with a surface albedo of 0 . Applying a larger surface albedo may lead to quantitatively different results. However, the qualitative differences will remain the same.

In general, the deviations from the 3 -D results increase with decreasing resolution. On the other hand, above a certain resolution, the errors of the ICA will in- crease again owing to the large contributions of horizontal transports which are associated with narrow atmospheric columns. It follows that there must exist a specific resolution which provides best results for domainaveraged fluxes. From the cloud resolutions used in this study, we can only conclude that this horizontal box size is about $1 \mathrm{~km}$. Furthermore, it should be noted that the good agreement between the ICA and the PPHOM approximation for small horizontal resolutions does not justify the latter because at the same time both the ICA and the PPHOM approximation deviate from the 3 -D results.

Local (column level) errors of the ICA due to the neglect of horizontal transports are largest at extreme solar zenith angles and at regions with strong horizontal gradients of the cloud optical thickness. For these conditions, the remote sensing of cloud properties (e.g., advanced very high resolution radiometer visible channel with $1-\mathrm{km}$ resolution at nadir) will provide largest errors if the remote sensing algorithms are based on plane-parallel homogeneous cloud geometries.

Finally, we note that the results of this study are based on a finite number of cloud realizations from a single atmospheric model with $1-\mathrm{km}$ (convective and 
stratified clouds) and 2-km (multilayered clouds) horizontal resolution. Therefore future work will consider cloud structures from other sources like large eddy simulations and cloud radar.

Acknowledgment. The work of R. Scheirer was supported by the GKSS Hochschulprogramm 5.T4.00 G01-HS1.

\section{References}

Bucholtz, A., Rayleigh-scattering calculations for the terrestrial atmosphere, Appl. Opt., 34, 2765-2773, 1995.

Cahalan, R. F., W. Ridgway, and W. J. Wiscombe, Independent pixel and Monte Carlo estimates of stratocumulus albedo, J. Atmos. Sci., 51, 3776-3790, 1994.

Chambers, L. H., A. Wielicki, and K. F. Evans, Independent pixel and two-dimensional estimates of Landsat-derived cloud field albedo, J. Atmos. Sci., 54, 1525-1532, 1997.

Chandrasekhar, S., Radiative Transfer, 393 pp., Dover, Mineola, N. Y., 1960.

Charache, D. H., V. J. Abreu, W. R. Kuhn, and W. R. Skinner, Incorporation of multiple cloud layers for ultraviolet radiation modeling studies, J. Geophys. Res., 99, 23,031-23,039, 1994.

Eppel, D. P., H. Kapitza, M. Clausen, D. Jacob, W. Koch, W. Levkov, H.-T. Mengelkamp, and N. Werrmann, The non-hydrostatic mesoscale model GESIMA, Part II, Parameterizations and applications, Contrib. Atmos. Phys., 68, 15-41, 1998.

Fu, Q., M. C. Cribb, H. W. Barker, S. K. Krueger, and A. Grossmann, Cloud geometry effects on atmospheric solar absorption, J. Atmos. Sci., 57, 1156-1168, 2000.

Hagedorn, R., Hydrologiebilanz im Geesthachter Simulationsmodell der Atmosphäre (GESIMA) als Test der mikrophysikalischen Parametrisierung, master's thesis, 81 pp., Math.-Naturwiss. Fakultät der Christian-AlbrechtsUniv. zu Kiel, Kiel, Germany, 1996.

Levkov, L., B. Rockel, H. Kapitza, and E. Raschke, 3D mesoscale numerical studies of cirrus and stratus clouds by their time and space evolution, Contrib. Atmos. Phys., 65, 35-58, 1992.

Macke, A., J. Mueller, K. Nagel, and R. Stuhlmann, A cellular automaton model for cloud formation, in IRS96: Current Problems in Atmospheric Radiation, edited by W. L. Smith and K. Stamnes, pp. 234-237, A. Deepak, Hampton, Va., 1997.

Macke, A., D. L. Mitchell, and L. V. Bremen, Monte Carlo radiative transfer calculations for inhomogeneous mixed phase clouds, Phys. Chem. Earth, Ser. B, 24(3), 237-241, 1999.

Meerkötter, R., and M. Degünther, Three dimensional cloud effects and satellite UV mapping, $D L R$ Rep. 127, Inst. für Phys. der Atmos., Oberpfaffenhofen, Germany, 1999.

Rothman, L. S., et al., The HITRAN database: 1986 edition, Appl. Opt., 26, 4058-4097, 1987.

Scheirer, R., and A. Macke, Influence of the gaseous atmosphere on solar fluxes of inhomogeneous clouds, Phys. Chem. Earth., Ser. B, 25(2), 73-76, 2000.

Thekaekara, M. P., Extraterrestrial solar spectrum, 3000$6100 \AA$ at $1-\AA$ intervals, Appl. Opt., 13, 518-522, 1974.

A. Macke, and R. Scheirer, Institute for Marine Research, Department of Oceancirculation and Climate, Duesternbrooker Weg 20, D-24105 Kiel, Germany. (amacke@ifm.unikiel.de; rscheirer@ifm.uni-kiel.de)

(Received September 18, 2000; revised January 17, 2001; accepted January 23, 2001.) 